Journal of Information \& Knowledge Management, Vol. 6, No. 4 (2007) 303-304

(c) iKMS \& World Scientific Publishing Co.

\title{
AUTHOR INDEX Volume 6
}

A.-B. Abdel-Moteleb \& M. Woodman

Towards a Knowledge Management

System Development Method:

Critique of Some Relevant Theories and Methodologies

R. Alhajj see Kianmehr, K.

M. Z. Anis, A. Mukherjee \& B. K. Roy Application of Data Mining Techniques for Proper Design of Knowledge Repository

C. D. Blades see Randles, T. J.

N. Bontis see Trevinyo-Rodríguez, R. N.

R. Carty \& S. Walsh The Role of Middle Managers in Knowledge Transfer

A. Celjo see Handzic, M.

B. Cheuk Applying Snowden's

Narrative Technique to Conduct Project Debrief Within the British Council: An Exemplar of Knowledge Management Project

B. Cheuk Applying Sense-making and Narrative Techniques to Capture Lessons Learnt

C. F. Cheung, S. H. Fung, S. K. Kwok, W. B. Lee \& B. Tan A Study of Knowledge-Based Simulation for Enterprise Resources Planning

C. F. Cheung see Shek, W. Y.

S.-E. Cho, W. J. Lee \& J. Kim Classification of Knowledge Areas/Hierarchies and Its Implications in Mass Customisation: An Exploratory Study

S. Y. Choi see Kim, J. A.

Y. Y. Chong see Shek, W. Y.

Y.-C. Chung, C.-H. Tsai \& Y.-W. Hsu Research on the Correlation Among Critical Success Factors of Knowledge Management, Executive Degree of Knowledge Management Activities and New Product Development

Performance in Taiwan's High-Tech Firms

4 (2007) 261

K. Cormican \& L. Dooley Knowledge Sharing in a Collaborative Networked

1 (2007) 33

4 (2007) 251

3 (2007) 201

1 (2007) 25

3 (2007) 189

4 (2007) 297

3 (2007) 219

1 (2007) 1

3 (2007) 165

4 (2007) 241

4 (2007) 231

1 (2007) 45

2 (2007) 127

4 (2007) 231
Environment

Q. Ding \& W. PerrizoSupport-Less

Association Rule Mining Using Tuple Count Cube

L. Dooley see Cormican, K.

S. H. Fung see Cheung, C. F.

R. Gokakkar Effects of Social Identity

Processes on Coordination and

Knowledge Sharing in Geographically

Distributed Software Teams

M. Handzic, A. Lagumdzija \& A. Celjo A Survey of Knowledge Management Adoption in Public Administration

S. L. Hoe Organisational Learning: Conceptual Links to Individual Learning, Learning Organisation and Knowledge Management

Y.-W. Hsu see Chung, Y.-C.

A. Hunter see Lichtenstein, S.

R. Jung see Kim, J. A.

S. Kamoun-Chouk Environmental Scanning in Tunisian Manufacturing SMEs

K. Kianmehr, H. Zhang, K. Nikolov, T. Özyer \& R. Alhajj Utilising Neural Network and Support Vector Machine for Gene Expression Classification

E. H. Kim \& Y. Park Prediction of IS Project Escalation Based on Software Development Risk Management

J. A. Kim, S. Y. Choi \& R. Jung Agent Based Process Management

Environment - Mercury

J. Kim see Cho, S.-E.

S. K. Kwok see Cheung, C. F.

A. Lagumdzija see Handzic, M.

P. Lambe see Timbrell, G.

W. J. Lee see Cho, S.-E.

W. B. Lee see Shek, W. Y.
2 (2007) 105

$4(2007) 271$

2 (2007) 105

4 (2007) 241

4 (2007) 281

3 (2007) 219

3 (2007) 211

4 (2007) 261

2 (2007) 91

2 (2007) 127

1 (2007) 57

4 (2007) 251

2 (2007) 153

2 (2007) 127

1 (2007) 45

4 (2007) 241

3 (2007) 219

2 (2007) 139

1 (2007) 45

4 (2007) 231 
W. B. Lee see Cheung, C. F.

S. Lichtenstein, C. M. Parker \& A.

Hunter Dynamic Knowledge

Integration in Socio-Technical

Networks: An Interpretive Study of

Intranet Use for Knowledge

Integration

H. T. Loh see Zhan, J.

J. Meacham see Rauniar, R.

A. Mukherjee see Anis, M. Z.

K. Nikolov see Kianmehr, K.

L. O'Donnell see Royal, C.

T. Özyer see Kianmehr, K.

R. Palanisamy Capturing Users' Tacit Knowledge in ERP Implementation: An Exploratory Multi-Site Case Study

Y. Park see Kim, E. H.

C. M. Parker see Lichtenstein, S.

W. Perrizo see Ding, Q.

V. Perumal \& P. C. Woods The Need for Knowledge Management in the Malaysian Film Industry: A Case Study

T. J. Randles \& C. D. Blades Creating Explanations that Guide and Motivate

R. Rauniar, G. Rawski \& J. Meacham Collective Ambition, Creative Chaos, Information Redundancy, and Shared Knowledge in Integrated Product Development - Case Study

G. Rawski see Rauniar, R.
4 (2007) 241

2 (2007) 91

3 (2007) 181

2 (2007) 79

3 (2007) 201

4 (2007) 251

2 (2007) 115

4 (2007) 251

1 (2007) 9

2 (2007) 153

2 (2007) 91

4 (2007) 271

3 (2007) 173

1 (2007) 25

2 (2007) 79

2 (2007) 79
B. K. Roy see Anis, M. Z.

C. Royal \& L. O'Donnell Education for Sustainability: Creating Skills in Human Capital Analysis

3 (2007) 201

$2(2007) 115$

W. Y. Shek, C. F. Cheung, W. B. Lee \& Y. Y. Chong Systematic Knowledge Auditing: A Case Study in a Power Utility Company

K. Stauss Exploring Knowledge Management and Knowledge Sharing in a Non-Profit Human Service Organisation

$\varnothing$. Taule, see Timbrell, G.

G. Timbrell, P. Lambe \& Ø. Taule Throwing Pebbles into a Dark Cave: A Study of Participation and Behaviour in the act-km Online Community

R. N. Trevinyo-Rodríguez \& N. Bontis The Role of Intellectual Capital in Mexican Family-Based Businesses: Understanding their Soul, Brain and Heart

C.-H. Tsai see Chung, Y.-C.

S. Walsh see Carty, R.

M. Woodman see Abdel-Moteleb, A.-B.

P. C. Woods see Perumal, V.

J. Zhan \& H. T. LohUsing Latent Semantic Indexing to Improve the Accuracy of Document Clustering

H. Zhang see Kianmehr, K.
1 (2007) 69

4 (2007) 241

2 (2007) 139

2 (2007) 139

$4(2007) 231$

3 (2007) 189

4 (2007) 261

4 (2007) 297

1 (2007) 33

3 (2007) 173

3 (2007) 181

4 (2007) 251 